\title{
Radiographic Osteoarthritis Prevalence Over Ten Years After Anterior Cruciate Ligament Reconstruction
}

\author{
Authors \\ Tianwu Chen ${ }^{1 *}$, Shuang Wang ${ }^{2 *}$, Yunxia $\mathrm{Li}^{1}$, Chengchong $\mathrm{Ai}^{1}$, Fangyi Jiang ${ }^{1}$, Shiyi Chen ${ }^{1}$
}

\section{Affiliations}

1 Sports Medicine Department, Huashan Hospital Fudan University, Shanghai, China

2 Department of Biochemistry and Molecular Biology, Tongji Medical College, Huazhong University of Science and Technology, Wuhan, China

\author{
Key words \\ anterior cruciate ligament reconstruction, osteoarthritis, \\ long-term, meta-analysis
}

accepted 09.04.2019

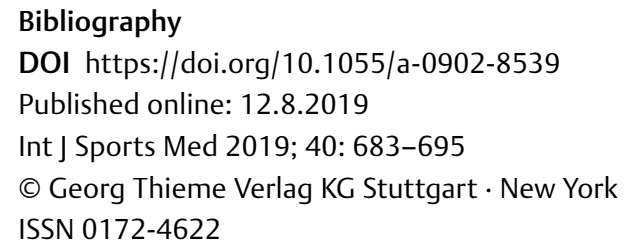

\section{Correspondence}

Prof. Shiyi Chen

Department Of Sports Medicine,

Huashan Hospital Fudan University,

No.433, Huashan Road,

Jingan District,

200020, Shanghai, China PR.,

Tel.: +86/138/01784 567, Fax: +86/021/52887 110

cshiyi@163.com

\begin{abstract}
The purpose of this study was to conduct an up-to-date systematic review and meta-analysis of radiographic knee osteoarthritis (OA) over minimal ten years after $A C L$ reconstruction. The database of Pubmed and the Ovid was adopted. The radiographic knee OA over minimal ten years after $A C L$ reconstruction was systematically reviewed. Both the ipsilateral and contralateral knees were evaluated referring to the tibiofemoral joint (TFJ), the patellofemoral joint (PFJ), and the overall knee OA prevalence. Nineteen studies were included for review, with nine screened for the meta-analysis. The overall knee OA rate ranged from $8.3-79.2 \%$, meanly $51.6 \%$ on the ipsilateral side; ranged from $3.6-35.7 \%$, meanly $15.5 \%$ on the contralateral side. Compared to the contralateral side, the RR of developing radiographic $\mathrm{OA}$ was $3.73(\mathrm{P}<0.01)$ for the overall knee, $2.88(P<0.01)$ for TFJ, and $2.42(P<0.01)$ for $P F J$. Ipsilaterally, the RR of developing TFJ radiographic OA was $1.15(\mathrm{P}<0.01)$ compared to that of the PFJ. Over a minimum of 10 years after surgery, more than half the cases developed overall radiographic OA on the ipsilateral knee, which was nearly four times higher than the contralateral side. On the ipsilateral knee, the TFJ was most affected.
\end{abstract}

\section{Introduction}

The long-term clinical outcomes of ACL reconstruction are richly reported in the literature [1-12]. Interestingly, the occurrence of radiographicosteoarthritis (OA) varies among those researchers, ranging from 10-90\% [13-16]. The inclusion of cases, evaluation methods, and follow-up period might be a good explanation for that inconsistency. Falciglia et al. reported follow-up research at meanly 13.6 years after $A C L$ reconstruction among the adolescent population [17], in which the radiographic OA rate was merely $8.3 \%$. While Lohmander et al. reported that the radiographic OA rate was $50 \%$ at $10-20$ years after ACL injury [13]. In contrast,
Gillquist et al. reported that rate could reach as high as over $70 \%$ at 15-20 years after ACL surgeries [18].

In 2009, Oiestad et al. systematically reviewed the rate of knee OA after $A C L$ injury [19], concluding that the prevalence of knee OA was low for isolated ACL injury and higher for subjects with combined injuries. However, the insufficient data from the included studies rendered it difficult to reach a firm conclusion on the prevalence of knee OA for more than 10 years. In 2013, Claes et al. reported a meta-analysis of knee radiographic OA after ACL surgery [20], suggesting that the knee OA prevalence after $A C L$ reconstruction is lower than commonly perceived. The same year, Ajuied et al. 
reported the other meta-analysis on this topic [21], concluding that $\mathrm{ACL}$ reconstruction had a role in reducing the risk of developing degenerative changes at 10 years. Remarkably, in the meta-analysis abovementioned, only a limited number of studies were included. Additionally, the evidence quality was lower with many as retrospective cohorts or case series, which inevitably affect the efficacy of conclusion.

During the past several years, studies concerning the long-term clinical outcomes of the $A C L$ reconstruction were successively reported $[1,6,7,17,22-34]$. Some were randomized control trials or prospective cohort studies [1, 22, 23, 26, 27, 29, 34] of relatively higher evidence quality compared with previous reports. Due to standardized reporting strategy and better design, more detailed OA information could be attained, including those of the ipsilateral and contralateral sides, as well as those of the tibiofemoral joint (TFJ) and patellofemoral joint (PFJ). Over the long term after $\mathrm{ACL}$

- Table 1a Searching Strategy and Outcomes from PubMed.

\begin{tabular}{|c|l|r|}
\hline Search Step & Search Terms & Number \\
\hline 1 & Anterior cruciate ligament [MeSH] & 9869 \\
\hline 2 & Knee joint [MeSH] & 52908 \\
\hline 3 & Ligaments, articular [MeSH] & 27786 \\
\hline 4 & Knee joint OR Ligaments, articular & 73171 \\
\hline 5 & Knee injuries [MeSH] & 22325 \\
\hline 6 & Osteoarthritis, knee [MeSH] & 15539 \\
\hline 7 & Osteoarthritis [MeSH] & 54274 \\
\hline 8 & Epidemiologic studies [MeSH] & 2120577 \\
\hline 9 & Epidemiology & 2124510 \\
\hline 10 & 2 or 7 & 97604 \\
\hline 11 & 1 or 4 or 5 & 81314 \\
\hline 12 & 6 or 10 & 97604 \\
\hline 13 & 8 or 9 & 3536969 \\
\hline 14 & 11 and 12 and 13 & 13564 \\
\hline 15 & Limits: humans, English & 12014 \\
\hline
\end{tabular}

- Table 1b Searching Strategy and Outcomes from Ovid.

\begin{tabular}{|c|l|r|}
\hline Search Step & Search Terms & Number \\
\hline 1 & ligaments or ligaments, articular & 122764 \\
\hline 2 & Anterior Cruciate Ligament & 66387 \\
\hline 3 & Knee Joint & 128822 \\
\hline 4 & Knee Injuries & 27067 \\
\hline 5 & Osteoarthritis & 321405 \\
\hline 6 & Osteoarthritis, Knee & 27003 \\
\hline 7 & Epidemiology & 2388942 \\
\hline 8 & Epidemiologic studies & 126831 \\
\hline 9 & 7 or 8 & 2477116 \\
\hline 10 & 3 or 5 & 418964 \\
\hline 11 & 1 or 3 & 241184 \\
\hline 12 & 2 or 4 or 11 & 297730 \\
\hline 13 & 6 or 10 & 418964 \\
\hline 14 & 9 and 12 and 13 & 3458 \\
\hline 15 & Limit 14 to English language & 3297 \\
\hline 16 & Limit to humans & 3248 \\
\hline
\end{tabular}

reconstruction, the OA development could be observed in both the TFJ and PFJ. Although the OA rate was as anticipated a higher level in the TFJ, it was also reported that the OA of the PFJ had been under-recognized, which could be prevalent after ACLR and was associated with worse symptoms and function [25, 35]. Considering the differences of clinic manifestation, treating methods, as well as the etiology between the OA of the TFJ and the PFJ, a meta-analysis of the OA development between the TFJ and PFJ could be meaningful for clinicians to better recognize the OA development in the long term after ACLR. So far, no investigation has compared the radiographic OA of the TFJ with that of the PFJ by meta-analysis. In addition, no study ever compared the radiographic OA of TFJ or PFJ between the ipsilateral and contralateral sides.

The purpose of this study was to conduct an up-to-date systematic review and meta-analysis of the knee radiographic OA over a minimum of ten years after $A C L$ reconstruction. Both the ipsilateral and contralateral knees were evaluated referring to the TFJ, the $\mathrm{PFJ}$, and the overall knee OA prevalence.

\section{Materials and Methods}

The systematic review was initiated on December 12, 2017. The retrieval platform included the Pubmed and the Ovid. The database included the Medline (since inception to December 12, 2017), the Embase (since 1974 to December 12, 2017), the Global Health Archive (from 1910 to 1972). The searching strategy used in the Pubmed and the Ovid was presented in > Tables $\mathbf{1} \mathbf{a}, \mathbf{b}$. For all returned studies, the titles and abstracts were respectively reviewed and assessed by inclusion and exclusion criteria, presented in > Table 2. If the titles and abstracts provided vague or insufficient information, the full texts were then reviewed. The references of included studies were reviewed for additional sources. The reviewing and screening process was respectively fulfilled by two investigators. Given disagreement on including or excluding of study, the discussion was required. If necessary, a professor specialized in sports medicine was invited to join the discussion. The registration number of the study is CRD42018084786. The study meets the ethical standards of Harriss et al. [36].

\section{Study Quality Assessment}

The Modified Coleman Methodology Score (MCMS) was applied for study quality evaluation. The original CMS was derived from the Consolidated Standards Of Reporting Trials (CONSORT) statement and applied for the review of patellar and Achilles tendinopathy [37, 38]. In 2009, Oiestad modified the CMS for cohort studies, and adopted it in reviewing the long-term radiographic $\mathrm{OA}$ after $\mathrm{ACL}$ injuries [19]. In the modified CMS, questions 2, 3, 6, and 7 in part A were altered or removed, resulting in a maximum score of 50 ; question 1 , criterion of sensitivity and the reliability of radiographic assessment in part $B$ were altered or removed, resulting in a maximum score of 40 . Therefore the maximum score of the modified CMS was 90. In this study, we adopted the same version as Oiestad et al. We compared the MCMS of the studies of different designs, published before and after December 31, 2013, and included it or did not include it in the meta-analysis. 
- Table 2 Study Selection Criteria.

\begin{tabular}{|c|c|}
\hline Inclusion criteria & Exclusion criteria \\
\hline 1. Prospective or retrospective design & 1. Follow-up period less than ten years \\
\hline 2. Patients with $\mathrm{ACL}$ tear surgically treated & 2. No radiographic exam performed \\
\hline 3. ACL reconstruction (Arthroscopically, Extra-articular and Intra-articular, Open surgery) & 3. No Kellgren\&Lawrence classification adopted \\
\hline 4. Radiographic exam at final follow-up & 4. No radiographic evaluation method was adopted \\
\hline 5. Adopting the Kellgren\&Lawrence classification & 5. The study involved the same subjects \\
\hline $\begin{array}{l}\text { 6. Isolate } \mathrm{ACL} \text { injury and } \mathrm{ACL} \text { injury combined with meniscal, cartilage, and medial } \\
\text { collateral ligament injuries }\end{array}$ & 6. No ACL reconstruction was performed \\
\hline 7. Minimal follow-up period of 10 years & \\
\hline 8. Written in English & \\
\hline
\end{tabular}

- Table 3 Inclusion criteria for meta-analysis.

\begin{tabular}{|cl|}
\hline \multicolumn{2}{|l|}{ Inclusion criteria } \\
\hline 1. & $\begin{array}{l}\text { Reporting the overall knee radiographic OA on both ipsilateral and } \\
\text { contralateral sides; }\end{array}$ \\
\hline 2. & $\begin{array}{l}\text { Reporting the TFJ radiographic OA on both ipsilateral and } \\
\text { contralateral sides; }\end{array}$ \\
\hline 3. & $\begin{array}{l}\text { Reporting the PFJ radiographic OA on both ipsilateral and } \\
\text { contralateral sides; }\end{array}$ \\
\hline 4. & Reporting the TFJ and the PFJ radiographic OA on the ipsilateral side; \\
\hline 5. & $\begin{array}{l}\text { Reporting the TFJ and the PFJ radiographic OA on the contralateral } \\
\text { side. }\end{array}$ \\
\hline
\end{tabular}

\section{Data extraction and statistical analysis}

The data were sorted and compiled with the Excel (Office 365, Microsoft). The authors, country, journal, study design, publish year, patients demographics, follow-up period, evaluation rate (subjects radiographically evaluated/subjects included), surgical technique, graft, meniscus injury, cartilage injury, radiographic outcomes, and position radiograph were extracted. The inclusion criteria for meta-analysis were presented in $>$ Table 3.

For the scarce number of randomized control studies that adopted the Kellgren\&Lawrence classification [1, 23, 29], this study included the non-randomized prospective cohort studies in metaanalysis. This method has been successfully adopted in previous studies [21,39].

The grade II of Kellgren\&Lawrence classification was set as the cut-off point for radiographic OA. The grading algorithm of the TFJ, the PFJ, and overall knee radiographic OA was consistent with previous reports. For unreported data necessitated for the meta-analysis, requesting emails were sent to the corresponding authors.

The Review Manager 5 (Revman) was adopted for the Preferred Reporting Items for Systematic Reviews and Meta-Analyses (PRISMA) and meta-analysis. The risk ratios (RR) with its $95 \%$ Confidential Interval $(\mathrm{Cl})$ were calculated based on the data extracted in the meta-analysis using the Review Manager 5 (Revman). The publication bias was visually inspected by funnel plots for asymmetry within the comparison of interest. The random effects model and fixed effects model were adopted for data pooling. The random effects model was used to reduce bias from systematic errors given higher heterogeneity of the included studies. Heterogeneity was quantified by the $\mathrm{I}^{2}$ statistic, with $\mathrm{P}<0.10$ being statistically significant. We performed a sensitivity analysis, in which one study was in turn removed at a time while the others were analyzed to estimate whether the $\mathrm{I}^{2}$ result could be affected markedly by the study removed.

\section{Results}

\section{General information}

A total of 15383 studies were obtained from the database. With all duplicates removed, 13685 articles were screened using the inclusion and exclusion criteria. Then 144 studies were identified and carefully read. Eventually, 19 articles were finally included in the systematic review $[1,4-6,8,16,17,22,27,29,31,34,40-46]$, and nine articles were included in the mate-analysis $[1,5,22,27,29$, 40, 41, 45, 46] (> Fig. 1).

For the articles included, 4 were randomized controlled trials $[1,22,34,41], 8$ were cohort studies [5, 27, 29, 31, 40,44-46], 5 were case series $[4,6,16,17,42]$, and 2 were case-control studies $[8,43]$. One group of subjects from a case-control study was not included because the follow-up period was less than ten years [43]. In the other case-control study, one group of subjects was excluded for conservative treatment [8]. The corresponding authors of four studies ( $26.7 \%$ response rate) replied to our request for unpublished data [1, 22, 27, 29].

\section{Study characteristics}

In the 19 articles included, the sample size ranged from 12 [17] to 210 [29]. A total of 1642 cases were included in the analysis, and 1,273 of these were radiographically evaluated, with evaluation rate ranging from $58.5 \%$ [34] to $100 \%[4,6,8,16,17,42]$. The follow-up period ranged from 10 years [5, 8, 42, 45] to 23 years [27], with a mean follow-up period of 15.4 years. Regarding the graft choice, the autologous hamstring (HT) was applied in all cases from four articles [6, 17, 27, 42]; the autologous bone patella tendon bone (BPTB) in all cases from seven articles [4, 8, 16, 41, 44-46]; the autologous HT or BPTB, respectively, in one group of cases from five articles [1, 5, 22, 29, 34]; synthetic ligament in some cases from two articles [31,40]; the BPTB allografts were applied in one group of cases from one article [43]. Arthroscopic single bundle ACL reconstruction was adopted in 18 articles. Among them, one group of cases from one article was treated additionally by extra-articular $\mathrm{ACL}$ reconstruction using iliotibial tract, namely the Macintosh technique modified by Cocker Arnold [27]; in another article, open 


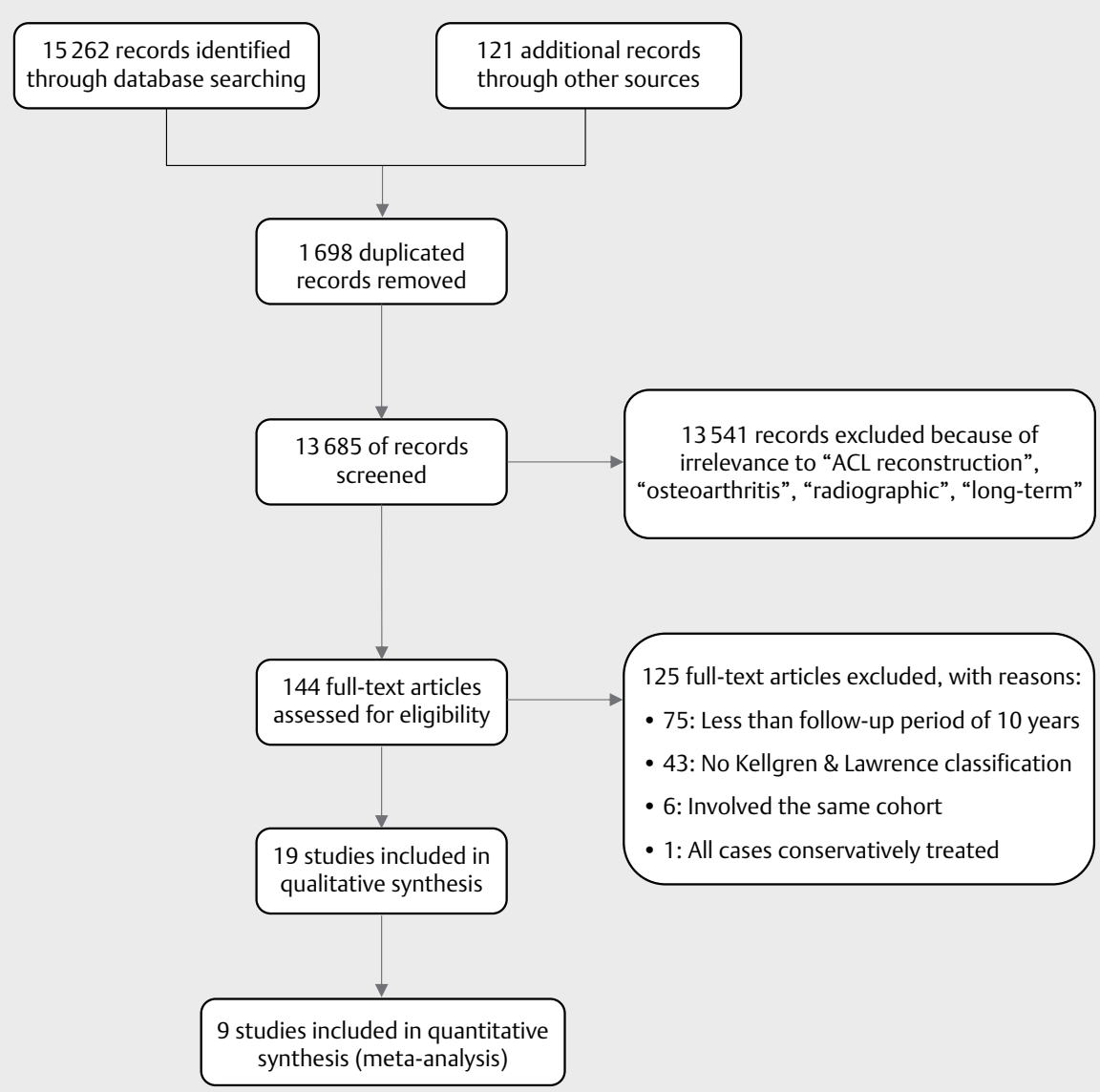

Fig. 1 PRISMA flow diagram of the search strategy.

ACL reconstruction was performed in one group of cases. The open $A C L$ reconstruction was performed in all cases in one article [41].

The details of combined meniscus injuries were not reported in 5 articles [4-6, 31, 45]. One article expelled the cases of combined meniscus injuries [16]. The remaining 13 articles described the details of combined meniscus injuries. The rate of combined injuries ranged from $16.7 \%$ [17] to $72.1 \%$ [42] with a mean rate of $49.5 \%$. One article expelled the cases of combined cartilage injuries; in addition, only five articles described the combined cartilage injuries. More details are presented in $>$ Tables $\mathbf{4 a}, \mathbf{b}$.

\section{Methodological Quality}

The details of the methodology used to evaluate the quality of the included studies, the studies with different designs, the studies published before and after December 31, 2013, and the studies included or not in the meta-analysis, were presented in > Tables 5a-d.

\section{Radiographic OA rate}

Sixteen articles reported the knee radiographic OA on the ipsilateral side $[1,4,5,8,16,17,22,27,31,34,40-42,44-46]$. Seven articles reported the knee radiographic $O A$ on the contralateral side $[1,5,22,40,41,45,46]$.
Four articles reported the radiographic OA of the ipsilateral TFJ $[22,27,29,40]$. Three articles reported the radiographic OA of the contralateral TFJ [22, 29, 40].

Four articles reported the radiographic OA of the ipsilateral PF] $[22,27,29,40]$. Three articles reported the radiographic OA of the contralateral PF] $[22,29,40]$. More details were presented in $>$ Tables 6a-e.

One study included in this study reported the OA rate of the TFJ and the PFJ, but failed in reporting the outcomes of the overall knee $\mathrm{OA}$ rate, resulting in the higher $\mathrm{OA}$ rate of TFJ and PFJ compared with that of the overall knee joint. The definition of radiographic OA was atypical in two studies $[6,43]$. One did not set the cut-off point for radiographic $O A$, simply concluding that the radiographic OA rate of the ipsilateral TFJ was significantly higher than the contralateral side [6]. The other one defined the radiographic OA as a side-to-side difference of Grade II or worse in 1 or more compartments or a side-to-side difference of Grade I in 2 or more compartments according to the Kellgren-Lawrence classification [43]. On that basis, the radiographic knee joint OA rate was $52.9 \%$.

\section{Meta-analysis}

A total of nine articles were eligible for meta-analysis [1, 5, 22, 27 , $29,40,41,45,46]$. Of these, seven compared the overall OA rate between ipsilateral and contralateral sides [1, 5, 22, 40, 41, 45, 46]; 


\begin{tabular}{|c|c|c|c|c|c|c|c|c|c|c|}
\hline 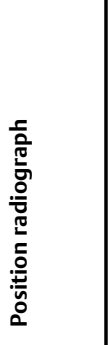 & 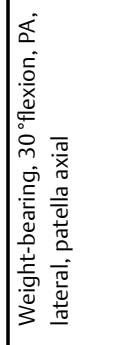 & 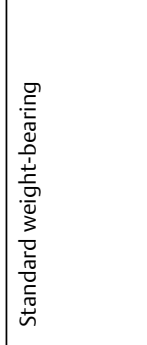 & 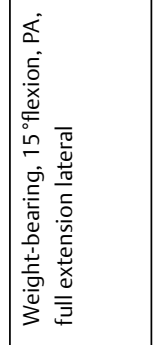 & 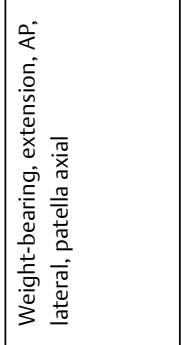 & 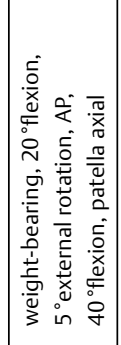 & $\frac{\pi}{2}$ & 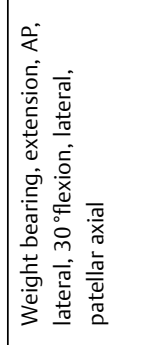 & $\frac{\pi}{z}$ & 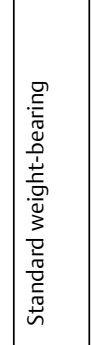 & 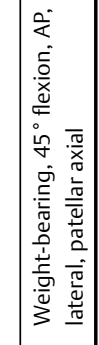 \\
\hline 区 & 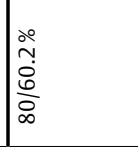 & 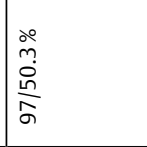 & 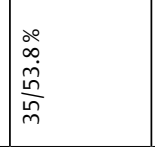 & 驾 & 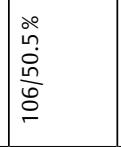 & 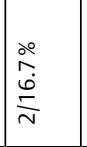 & 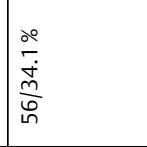 & $\frac{\frac{\pi}{2}}{\frac{\pi}{2}}$ & $\mid \frac{\frac{\pi}{2}}{\frac{\pi}{z}}$ & 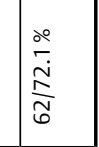 \\
\hline 蒙 & 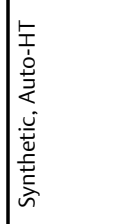 & 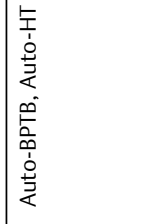 & 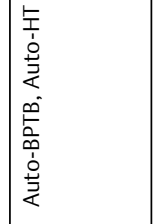 & \begin{tabular}{|l} 
t古 \\
妾
\end{tabular} & 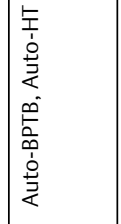 & 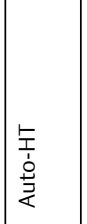 & 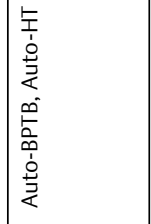 & 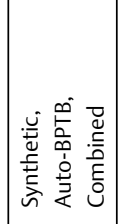 & 章 & $\begin{array}{l}\text { 幹 } \\
\text { 妾 }\end{array}$ \\
\hline 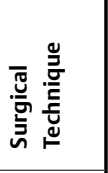 & 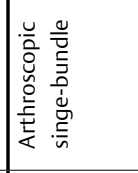 & 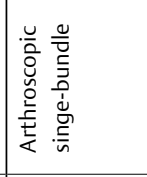 & 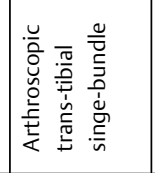 & 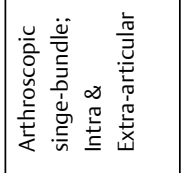 & 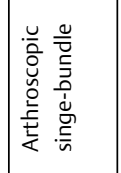 & 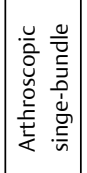 & 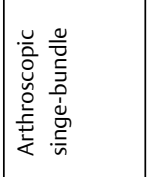 & 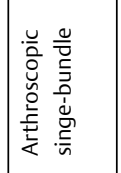 & 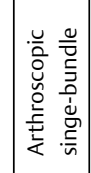 & 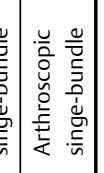 \\
\hline 总 & 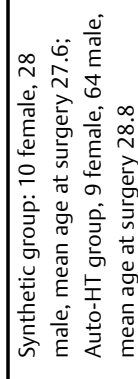 & 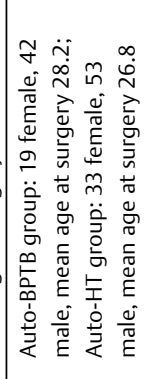 & 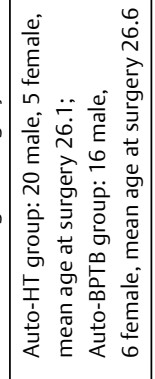 & 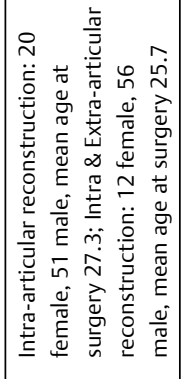 & 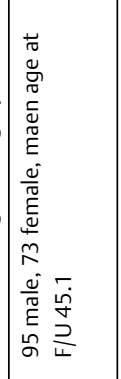 & 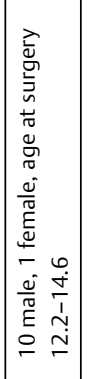 & 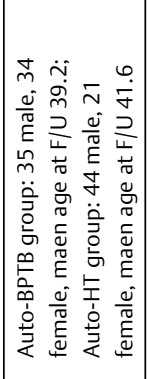 & 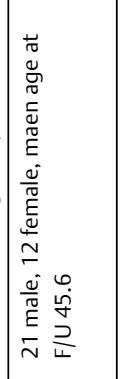 & 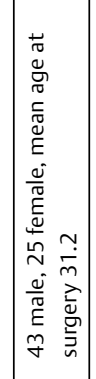 & 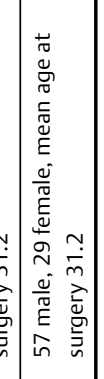 \\
\hline 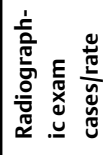 & 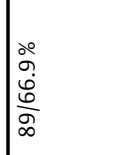 & 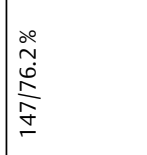 & 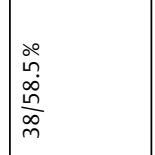 & 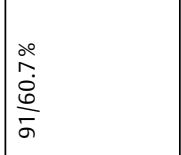 & 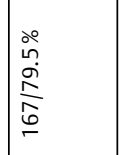 & 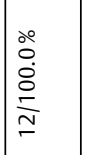 & 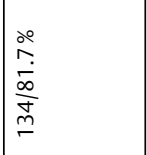 & $\frac{x}{\frac{x}{2}}$ & 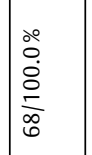 & 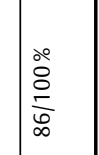 \\
\hline 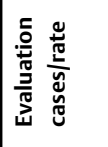 & 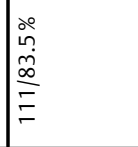 & 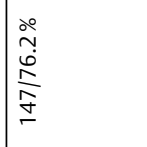 & 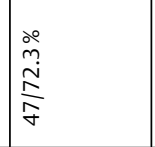 & 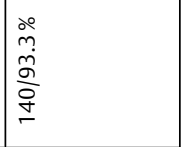 & 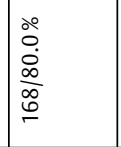 & 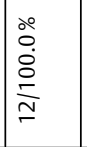 & 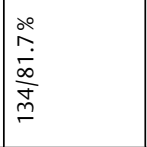 & 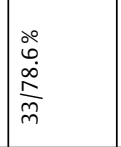 & 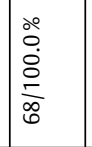 & 递 \\
\hline 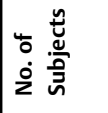 & $\stackrel{m}{\underline{m}}$ & $\stackrel{\varrho}{\underline{g}}$ & 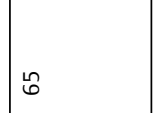 & 号 & $\stackrel{\circ}{i}$ & $\simeq$ & $\Phi$ & f & $\infty$ & $\infty$ \\
\hline 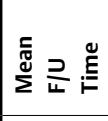 & $\stackrel{\simeq}{0}$ & 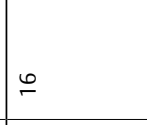 & $\stackrel{m}{\underline{\rho}}$ & 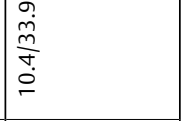 & $\stackrel{\infty}{\stackrel{\infty}{\digamma}}$ & $\stackrel{+}{\stackrel{+}{\circ}}$ & $\bar{g}$ & $\approx$ & 吕 & $\because$ \\
\hline 咅 & 品 & 离 & 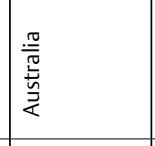 & 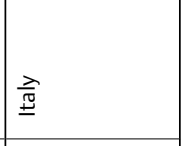 & 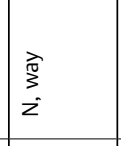 & 暜 & 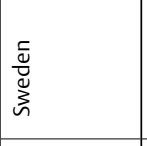 & \begin{tabular}{|l} 
\\
离 \\
离
\end{tabular} & 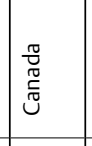 & 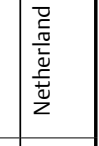 \\
\hline 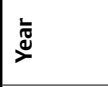 & $\overline{\bar{i}}$ & 产 & 产 & 号 & 产 & 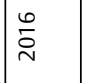 & 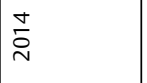 & 商 & 产 & $\stackrel{m}{\grave{N}}$ \\
\hline 蒿 & 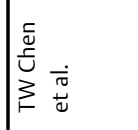 & 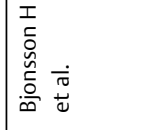 & 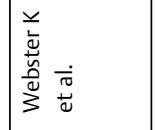 & 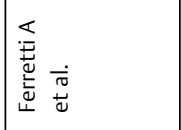 & 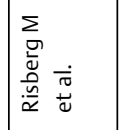 & 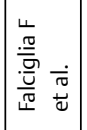 & 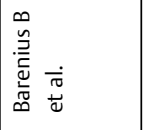 & 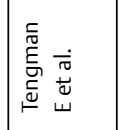 & 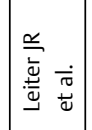 & 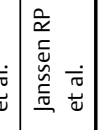 \\
\hline
\end{tabular}




\begin{tabular}{|c|c|c|c|c|c|c|c|c|c|}
\hline 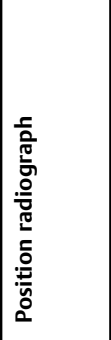 & 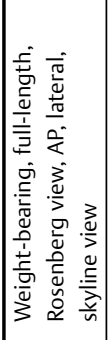 & 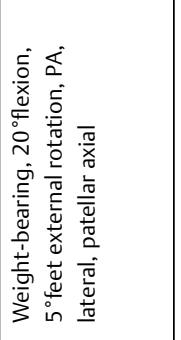 & 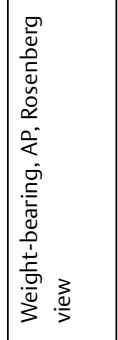 & - & 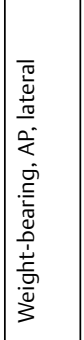 & 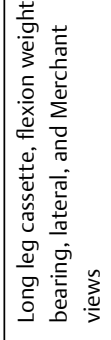 & 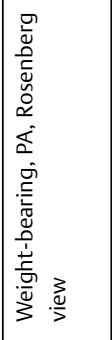 & 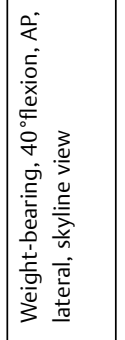 & 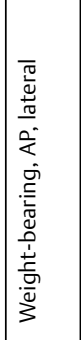 \\
\hline 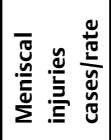 & $\underline{z}$ & 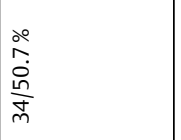 & $\Sigma$ & \begin{tabular}{|l}
$\bar{d}$ \\
$\frac{\vec{v}}{\bar{y}}$ \\
$\bar{x}$
\end{tabular} & $\Sigma$ & $\begin{array}{l}\stackrel{\circ}{N} \\
\tilde{N} \\
\stackrel{0}{N} \\
\end{array}$ & 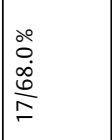 & 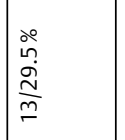 & 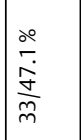 \\
\hline 营 & 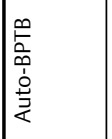 & 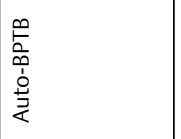 & 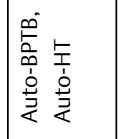 & 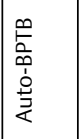 & 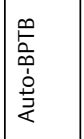 & 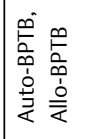 & 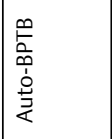 & 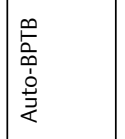 & 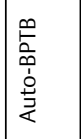 \\
\hline 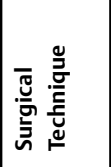 & 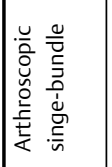 & 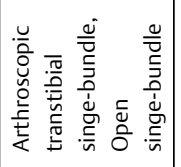 & 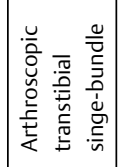 & 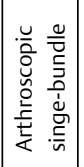 & 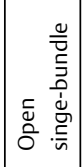 & 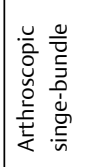 & 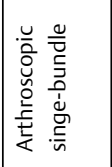 & 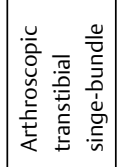 & 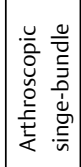 \\
\hline 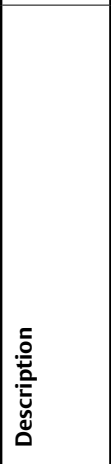 & 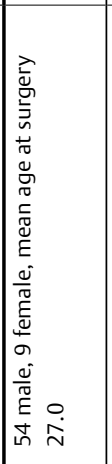 & 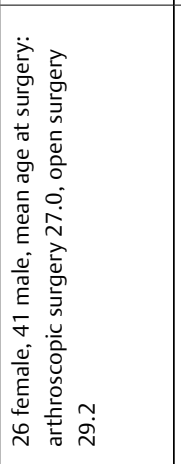 & 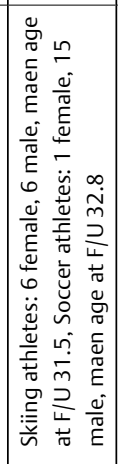 & 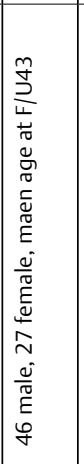 & 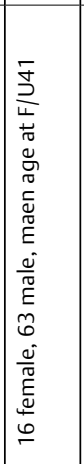 & 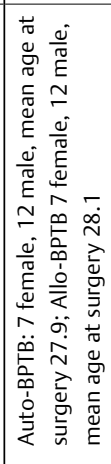 & 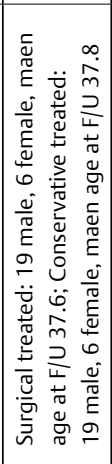 & 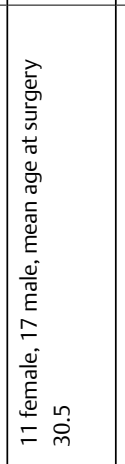 & 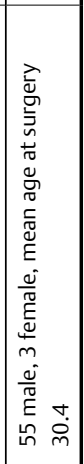 \\
\hline 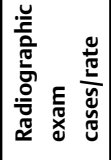 & 产 & $\frac{\stackrel{\circ}{\circ}}{\frac{\rho}{\hat{N}}}$ & 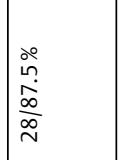 & $\frac{\stackrel{\circ}{o}}{\frac{\partial}{m}}$ & 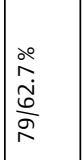 & $\begin{array}{l}\stackrel{\circ}{2} \\
\text { in } \\
\infty \\
\frac{\infty}{\Sigma} \\
\end{array}$ & $\begin{array}{l}\stackrel{\circ}{\circ} \\
\stackrel{\circ}{\grave{n}}\end{array}$ & 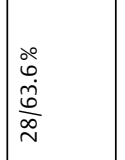 & 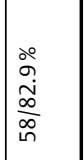 \\
\hline 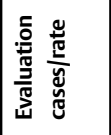 & $\frac{\stackrel{\circ}{\circ}}{\frac{\partial}{\hat{\theta}}}$ & $\frac{\stackrel{\partial}{\circ}}{\frac{\dot{\rho}}{M}}$ & 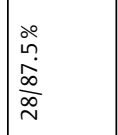 & $\frac{\stackrel{\circ}{o}}{\frac{0}{m}}$ & $\begin{array}{l}\frac{o}{\hat{i}} \\
\frac{1}{2} \\
\frac{2}{2}\end{array}$ & 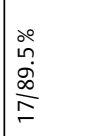 & $\begin{array}{l}\stackrel{\circ}{\circ} \\
\stackrel{\circ}{\grave{n}}\end{array}$ & $\begin{array}{l}\stackrel{\circ}{6} \\
\tilde{\omega} \\
\stackrel{0}{\infty} \\
\stackrel{\infty}{N}\end{array}$ & $\begin{array}{l}\stackrel{\circ}{\circ} \\
\stackrel{\infty}{\infty} \\
\infty \\
\infty \\
\infty\end{array}$ \\
\hline 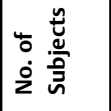 & $\tilde{\theta}$ & 6 & $\approx$ & $\stackrel{m}{\wedge}$ & $\stackrel{\stackrel{\stackrel{\sim}{ }}{\simeq}}{ }$ & 9 & $\stackrel{\leftrightarrow}{\sim}$ & F & 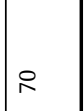 \\
\hline 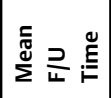 & $\stackrel{0}{\circ}$ & $\simeq$ & $\stackrel{\circ}{\circ}$ & $\stackrel{\stackrel{m}{m}}{\stackrel{m}{m}}$ & $\stackrel{\circ}{\circ}$ & 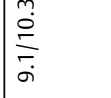 & $\circ$ & $\stackrel{m}{\rho}$ & $\stackrel{\stackrel{\sim}{=}}{=}$ \\
\hline $\begin{array}{l}\text { È } \\
\stackrel{E}{5}\end{array}$ & 竞 & 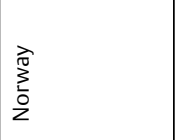 & 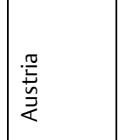 & 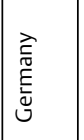 & 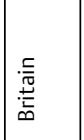 & 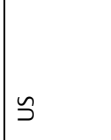 & 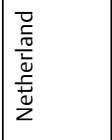 & 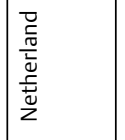 & 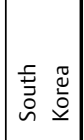 \\
\hline 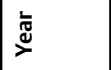 & $\stackrel{m}{\stackrel{m}{\nu}}$ & $\stackrel{\sim}{\tilde{\nu}}$ & $\stackrel{\sim}{\stackrel{\sim}{\sim}}$ & $\stackrel{\tilde{D}}{\sim}$ & $\stackrel{\circ}{\stackrel{0}{\sim}}$ & 号 & : & $\stackrel{\infty}{\stackrel{\infty}{े}}$ & $\underset{\sim}{\stackrel{0}{े}}$ \\
\hline 贶 & 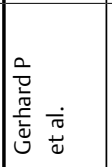 & 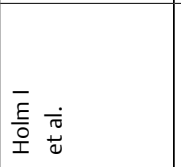 & 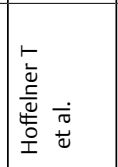 & 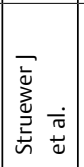 & 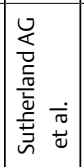 & 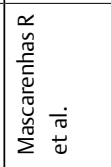 & 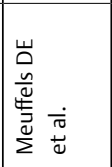 & 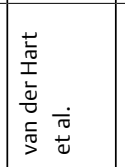 & 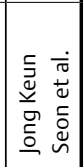 \\
\hline
\end{tabular}


- Table 5a Modified Coleman Methodology Score (MCMS) of the studies included.

\begin{tabular}{|c|c|c|c|c|c|c|}
\hline Author & Meta-analysis & Design & Year & MCMSA & MCMSB & MCMS \\
\hline TW Chen et al. & Ipsilateral vs. contralateral TFJ vs. PFJ & Cohort & 2017 & 48 & 38 & 86 \\
\hline Bjornsson $\mathrm{H}$ et al. & Ipsilateral vs. contralateral & RCT & 2016 & 40 & 33 & 73 \\
\hline van der Hart et al. & Ipsilateral vs. contralateral & Cohort & 2008 & 24 & 24 & 48 \\
\hline Barenius B et al. & Ipsilateral vs. contralateral TFJ vs. PFJ & RCT & 2014 & 30 & 40 & 70 \\
\hline Holm I et al. & Ipsilateral vs. contralateral & RCT & 2012 & 44 & 30 & 73 \\
\hline Sutherland AG et al. & Ipsilateral vs. contralateral & Cohort & 2010 & 28 & 32 & 60 \\
\hline Hoffelner T et al. & Ipsilateral vs. contralateral & Cohort & 2012 & 24 & 29 & 53 \\
\hline Ferretti A et al. & TFJ vs. PFJ & Cohort & 2016 & 33 & 30 & 63 \\
\hline Risberg M et al. & TFJ vs. PFJ & Cohort & 2016 & 38 & 38 & 76 \\
\hline Falciglia F et al. & Not included & Case series & 2016 & 25 & 13 & 38 \\
\hline Webster $\mathrm{K}$ et al. & Not included & RCT & 2016 & 44 & 26 & 70 \\
\hline Struewer J et al. & Not included & Case series & 2012 & 35 & 18 & 53 \\
\hline Mascarenhas R et al. & Not included & Case control & 2010 & 24 & 30 & 54 \\
\hline Meuffels DE et al. & Not included & Case control & 2009 & 27 & 37 & 64 \\
\hline Seon JK et al. & Not included & Cohort & 2006 & 32 & 25 & 57 \\
\hline Tengman E et al. & Not included & Cohort & 2014 & 48 & 19 & 67 \\
\hline Leiter JR et al. & Not included & Case series & 2014 & 33 & 26 & 59 \\
\hline Janssen RP et al. & Not included & Case series & 2013 & 28 & 29 & 57 \\
\hline Gerhard P et al. & Not included & Case series & 2013 & 35 & 20 & 55 \\
\hline
\end{tabular}

- Table 5b Modified Coleman Methodology Score (MCMS) of studies included with different designs.

\begin{tabular}{|l|l|l|l|l|}
\hline Study Design & $\begin{array}{l}\text { No. of } \\
\text { articles }\end{array}$ & $\begin{array}{l}\text { MCMSA } \\
\text { (Mean, } \\
\text { range) }\end{array}$ & $\begin{array}{l}\text { MCMSB } \\
\text { (Mean, } \\
\text { range) }\end{array}$ & $\begin{array}{l}\text { MCMS } \\
\text { (Mean, } \\
\text { range) }\end{array}$ \\
\hline RCT & 4 & $40 ; 30-44$ & $32 ; 26-40$ & $72 ; 70-73$ \\
\hline Cohort & 8 & $34 ; 24-48$ & $29 ; 19-38$ & $64 ; 48-86$ \\
\hline Case control & 2 & $26 ; 24-27$ & $34 ; 30-37$ & $59 ; 54-64$ \\
\hline Case series & 5 & $31 ; 25-35$ & $21 ; 13-29$ & $52 ; 38-59$ \\
\hline
\end{tabular}

- Table 5c Modified Coleman Methodology Score (MCMS) of studies published before and after the $31^{\text {st }}$ December 2013.

\begin{tabular}{|l|c|l|l|l|}
\hline $\begin{array}{l}\text { Publication } \\
\text { Year }\end{array}$ & $\begin{array}{l}\text { No. of } \\
\text { articles }\end{array}$ & $\begin{array}{l}\text { MCMSA } \\
\text { (Mean, } \\
\text { range) }\end{array}$ & $\begin{array}{l}\text { MCMSB } \\
\text { (Mean, } \\
\text { range) }\end{array}$ & $\begin{array}{l}\text { MCMS } \\
\text { (Mean, } \\
\text { range) }\end{array}$ \\
\hline $2014-2017$ & 9 & $38 ; 25-48$ & $29 ; 13-40$ & $67 ; 38-86$ \\
\hline $2006-2013$ & 10 & $30 ; 24-44$ & $27 ; 18-37$ & $57 ; 48-73$ \\
\hline
\end{tabular}

- Table 5d Modified Coleman Methodology Score (MCMS) of the studies included/not included in the meta-analysis.

\begin{tabular}{|l|c|l|l|l|}
\hline Meta-analysis & $\begin{array}{l}\text { No. of } \\
\text { articles }\end{array}$ & $\begin{array}{l}\text { MCMSA } \\
\text { (Mean, } \\
\text { range) }\end{array}$ & $\begin{array}{l}\text { MCMSB } \\
\text { (Mean, } \\
\text { range) }\end{array}$ & $\begin{array}{l}\text { MCMS } \\
\text { (Mean, } \\
\text { range) }\end{array}$ \\
\hline $\begin{array}{l}\text { Ipsilateral Vs } \\
\text { contralateral }\end{array}$ & 7 & $34 ; 24-48$ & $32 ; 24-40$ & $66 ; 48-86$ \\
\hline TFJ Vs PFJ & 4 & $37 ; 30-48$ & $37 ; 30-40$ & $74 ; 63-86$ \\
\hline Not included & 10 & $33 ; 24-48$ & $24 ; 13-37$ & $57 ; 38-70$ \\
\hline
\end{tabular}

three compared the TFJ OA rate between ipsilateral and contralateral sides [22, 29, 40]; three compared the PFJ OA rate between ipsilateral and contralateral sides [22, 29, 40]; four compared the TF] OA with the PFJ OA on the ipsilateral side [22, 27, 29, 40]; three compared the TFJ OA with the PFJ OA on the contralateral side $[22,29,40]$. The extracted data was insufficient to perform the meta-analysis relating to the combined meniscus and cartilage injuries. ( $\triangleright$ Table $6 g$ )

Compared with contralateral control, the risk ratio of developing overall knee radiographic OA was 3.73 (range, 2.66-5.22; $\mathrm{P}<0.00001$ ) on the ipsilateral side; the risk ratio of developing PFJ radiographic OA was 2.42 (range, $1.60-3.67$; $P<0.0001$ ) on the ipsilateral side; the risk ratio of developing TFJ radiographic OA was 2.88 (range, 2.15-3.87; $\mathrm{P}<0.00001$ ) on the ipsilateral side. Compared with the $\mathrm{PF}$, the risk ratio of developing radiographic $\mathrm{OA}$ in the TFJ was 1.15 (range, 1.03-1.28; $\mathrm{P}=0.01$ ) on the ipsilateral side. While on the contralateral side, the risk ratio was not statistically different between the PFJ and TFJ $(P=0.38)$. By the sensitivity analysis, the studies by Risberg, Barenius, and Hoffelner et al. were removed from meta-analysis. In all five meta-analyses, the heterogeneity of the $\mathrm{I}^{2}$ index ranged from 0-50\%. For details, - Fig. 2 a-e.

\section{Discussion}

The systematic review and meta-analyses of the radiographic OA rate at a minimum of ten years after $A C L$ reconstruction was performed in this study. For the radiographic $\mathrm{OA}$, in addition to the comparison between the ipsilateral and the contralateral side, the comparison between the tibiofemoral and the patellofemoral joint was also made.

In the systematic reviews and meta-analysis previously reported, researchers focused on the minimal ten-year knee OA rate after 
- Table 6a The Overall Knee Radiographic OA Rate of the Ipsilateral Knee from the Studies Included.

\begin{tabular}{|c|c|c|c|c|c|c|c|}
\hline \multirow{2}{*}{ Author } & \multirow{2}{*}{ Year } & \multicolumn{5}{|c|}{ Kellgren \& Lawrence Classification } & \multirow{2}{*}{ Radiographic OA rate } \\
\hline & & $\mathbf{0}$ & $\mathbf{I}$ & II & III & IV & \\
\hline Bjornsson H et al. & 2016 & 43 & 39 & 45 & 14 & 6 & $44.2 \%$ \\
\hline Ferretti A et al. & 2016 & 9 & 49 & 22 & 10 & 1 & $36.3 \%$ \\
\hline Barenius B et al. & 2014 & \multicolumn{5}{|c|}{83} & $61.9 \%$ \\
\hline TW Chen et al. & 2017 & 23 & 18 & 43 & 5 & 0 & $53.9 \%$ \\
\hline Tengman E et al. & 2014 & 2 & 5 & 12 & 10 & 4 & $78.8 \%$ \\
\hline Falciglia F et al. & 2016 & 7 & 4 & 1 & 0 & 0 & $8.3 \%$ \\
\hline Gerhard P et al. & 2013 & 8 & 30 & 5 & 9 & 4 & $32.1 \%$ \\
\hline Struewer J et al. & 2012 & 3 & 30 & 24 & 12 & 4 & $54.8 \%$ \\
\hline Janssen RP et al. & 2013 & 7 & 16 & 17 & 41 & 5 & $73.3 \%$ \\
\hline van der Hart et al. & 2008 & 3 & 12 & 8 & 3 & 2 & $46.4 \%$ \\
\hline Sutherland AG et al. & 2010 & \multicolumn{4}{|c|}{36} & 0 & $45.6 \%$ \\
\hline Hoffelner T et al. & 2012 & 19 & 2 & 1 & 2 & 4 & $24.1 \%$ \\
\hline Meuffles DE et al. & 2009 & 4 & 9 & 9 & 3 & 0 & $48.0 \%$ \\
\hline Seon JK et al. & 2006 & & 33 & 20 & 5 & 0 & $43.1 \%$ \\
\hline Webster K et al. & 2016 & & 11 & \multicolumn{3}{|c|}{27} & $28.9 \%$ \\
\hline Holm I et al & 2012 & 4 & 7 & 23 & 14 & 5 & $79.2 \%$ \\
\hline
\end{tabular}

- Table $6 \mathbf{b}$ The Overall Knee Radiographic OA Rate of the Contralateral Knee from the Studies Included.

\begin{tabular}{|c|c|c|c|c|c|c|c|}
\hline \multirow{2}{*}{ Author } & \multirow{2}{*}{ Year } & \multicolumn{5}{|c|}{ Kellgren \& Lawrence Classification } & \multirow{2}{*}{ Radiographic OA rate } \\
\hline & & 0 & 1 & II & III & IV & \\
\hline Bjornsson $\mathrm{H}$ et al. & 2016 & 117 & 16 & 9 & 0 & 1 & $7.0 \%$ \\
\hline Barenius B et al. & 2014 & \multicolumn{2}{|c|}{108} & \multicolumn{3}{|c|}{26} & $19.4 \%$ \\
\hline TW Chen et al. & 2017 & 46 & 25 & 18 & 0 & 0 & $20.2 \%$ \\
\hline van der Hart et al. & 2008 & 20 & 7 & 1 & 0 & 0 & $3.6 \%$ \\
\hline Sutherland AG et al. & 2010 & \multicolumn{2}{|c|}{73} & \multicolumn{3}{|c|}{6} & $7.6 \%$ \\
\hline Hoffelner T et al. & 2012 & 17 & 1 & 4 & 2 & 4 & $35.7 \%$ \\
\hline Holm I et al. & 2012 & 26 & 12 & 11 & 2 & 2 & $28.3 \%$ \\
\hline
\end{tabular}

- Table 6c The Tibiofemoral Joint Radiographic OA Rate of the Ipsilateral Knee from the Studies Included.

\begin{tabular}{|c|c|c|c|c|c|c|c|}
\hline \multirow{2}{*}{ Author } & \multirow{2}{*}{ Year } & \multicolumn{5}{|c|}{ Kellgren \& Lawrence Classification } & \multirow{2}{*}{ Radiographic $O A$ rate } \\
\hline & & 0 & $\mathbf{I}$ & II & III & IV & \\
\hline Ferretti A et al. & 2016 & 11 & 49 & 20 & 10 & 1 & $34.10 \%$ \\
\hline Risberg M et al. & 2016 & 10 & 12 & 99 & 35 & 11 & $86.80 \%$ \\
\hline Barenius B et al. & 2014 & \multicolumn{2}{|c|}{54} & \multicolumn{3}{|c|}{80} & $59.70 \%$ \\
\hline TW Chen et al. & 2017 & \multicolumn{2}{|c|}{45} & \multicolumn{3}{|c|}{44} & $49.40 \%$ \\
\hline
\end{tabular}

- Table 6d The Tibiofemoral Joint Radiographic OA Rate of the Contralateral Knee from the Studies Included.

\begin{tabular}{|c|c|c|c|c|c|c|c|}
\hline \multirow[t]{2}{*}{ Author } & \multirow[t]{2}{*}{ Year } & \multicolumn{5}{|c|}{ Kellgren \& Lawrence Classification } & \multirow{2}{*}{ Radiographic $O A$ rate } \\
\hline & & $\mathbf{0}$ & $\mathbf{I}$ & II & III & IV & \\
\hline Risberg M et al. & 2016 & 49 & 32 & 68 & 15 & 3 & $51.50 \%$ \\
\hline Barenius B et al. & 2014 & \multicolumn{2}{|c|}{109} & \multicolumn{3}{|c|}{25} & $18.70 \%$ \\
\hline TW Chen et al. & 2017 & \multicolumn{2}{|c|}{71} & \multicolumn{3}{|c|}{18} & $20.20 \%$ \\
\hline
\end{tabular}

ACL injury, while they did not exclude the cases conservatively treated [19-21]. In comparison, we only investigate the cases of $\mathrm{ACL}$ reconstruction in this study. For the portion of cases conservatively treated in some studies, they were specifically excluded from the analysis to minimize the heterogeneity of data.
The ipsilateral knee was affected more often than the contralateral side, and meanly over half proportion of the ACL reconstruction cases demonstrated radiographic degeneration a minimum of ten years after surgery. 
- Table 6e The Patellofemoral Joint Radiographic OA Rate of the Ipsilateral Knee from the Studies Included.

\begin{tabular}{|c|c|c|c|c|c|c|c|}
\hline \multirow{2}{*}{ Author } & \multirow{2}{*}{ Year } & \multicolumn{5}{|c|}{ Kellgren \& Lawrence Classification } & \multirow{2}{*}{ Radiographic $O A$ rate } \\
\hline & & 0 & I & II & III & IV & \\
\hline Ferretti A et al. & 2016 & 15 & 48 & 21 & 6 & 1 & $30.80 \%$ \\
\hline Risberg M et al. & 2016 & 13 & 20 & 123 & 10 & 1 & $80.20 \%$ \\
\hline Barenius B et al. & 2014 & \multicolumn{2}{|c|}{101} & \multicolumn{3}{|c|}{33} & $23.60 \%$ \\
\hline TW Chen et al. & 2017 & \multicolumn{2}{|c|}{64} & \multicolumn{3}{|c|}{25} & $28.10 \%$ \\
\hline
\end{tabular}

- Table 6f The Patellofemoral Joint Radiographic OA Rate of the Contralateral Knee from the Studies Included.

\begin{tabular}{|c|c|c|c|c|c|c|c|}
\hline \multirow[t]{2}{*}{ Author } & \multirow[t]{2}{*}{ Year } & \multicolumn{5}{|c|}{ Kellgren \& Lawrence Classification } & \multirow{2}{*}{ Radiographic $O A$ rate } \\
\hline & & $\mathbf{0}$ & $\mathbf{I}$ & II & III & IV & \\
\hline Risberg M et al. & 2016 & 46 & 27 & 89 & 5 & 0 & $56.30 \%$ \\
\hline Barenius B et al. & 2014 & \multicolumn{2}{|c|}{122} & \multicolumn{3}{|c|}{12} & $9.00 \%$ \\
\hline TW Chen et al. & 2017 & \multicolumn{2}{|c|}{75} & \multicolumn{3}{|c|}{14} & $15.70 \%$ \\
\hline
\end{tabular}

- Table 6g The Osteoarthritis Rates on Ipsilateral and Contralateral Sides.

\begin{tabular}{|l|c|l|}
\hline Position & No. of article & OA rate (Mean, range) \\
\hline Ipsilateral overall knee & 16 & $51.6 \%, 8.4 \%-79.2 \%$ \\
\hline Contralateral overall knee & 7 & $15.5 \%, 3.6 \%-35.7 \%$ \\
\hline Ipsilateral TFJ & 4 & $62.4 \%, 34.1 \%-86.8 \%$ \\
\hline Contralateral TFJ & 3 & $33.1 \%, 18.7 \%-51.5 \%$ \\
\hline Ipsilateral PFJ & 4 & $46.8 \%, 28.1 \%-80.2 \%$ \\
\hline Contralateral PFJ & 3 & $30.8 \%, 9.0 \%-56.3 \%$ \\
\hline
\end{tabular}

Ajuied et al. once performed a meta-analysis on the radiographic OA rate at a minimum of ten years after $A C L$ reconstruction [21]. In their study, the risk ratio of developing moderate to severe OA (grade III or IV, according to the Kellgren\&Lawrence classification) on the ipsilateral knee was 3.62 compared to that of the contralateral side. In this study, the risk ratio of developing overall radiographic OA ( $\geq$ grade II, according to the Kellgren\&Lawrence classification) on the ipsilateral knee was 3.73 compared to that of the contralateral side. The cut-off point was grade II, which was widely accepted in the radiographic degeneration evaluation using the Kellgren \& Lawrence classification. Among 19 studies included herein, 17 adopted grade II as the cut-off point for radiographic evaluation [1, 4, 5, 8, 16, 17, 20, 22, 27, 29, 31, 34, 41, 42, 44-46].

Harris et al. systematically reviewed the radiographic OA rate on the tibiofemoral joint long-term after $A C L$ injury that was either surgically or conservatively treated [47]. A total of 380 cases from four retrospective studies were investigated with a mean follow-up period of 11.8 years. The radiographic tibiofemoral OA rate on the ipsilateral knee ranged from 32.6-51.2\% for cases surgically treated, and ranged from $24.5-42.3 \%$ for cases conservatively treated. The researchers suggested that surgical treatment not be back by any definitive evidence to prevent tibiofemoral OA after ACL injury. In this study, the radiographic tibiofemoral OA rate ranged from $34.1-86.8 \%$ and was on average $62.4 \%$ for the ipsilateral knee. In contrast, it ranged from $18.7-51.5 \%$ and was a mean of $33.1 \%$ for the contralateral side. The risk ratio of developing tibiofemoral radiographic OA was 2.88 on the ipsilateral knee compared with that of the contralateral side. To the best of our knowledge, it was for the first time that the tibiofemoral radiographic OA rate between the ipsilateral and contralateral sides was meta-analyzed.

In the study reported by Risberg et al., the posttraumatic tibiofemoral OA was associated with combined meniscus injuries, while the extracted data in this study could not support further analysis about meniscal conditions [29].

The OA occurred in the PFJ was different from that in the TFJ regarding the clinic manifestation, treating methods, as well as the etiology. Although it was anticipated that risk of OA was higher in the TFJ for the nature of ACL tear, an injury mostly involving the other structures between the tibia and femur menisci and subchondral bones, the results were inconsistent in literature. Oiestad et al. reported the $\mathrm{PFJ} O A$ rate on the ipsilateral knee long-term after $A C L$ reconstruction [48]. Their study involved 210 cases with a mean follow-up period of 12.3 years, and the $\mathrm{PFJ}$ radiographic OA rate was $26 \%$. While in a narrative review by Culvenor et al., the researchers concluded that PFJ OA might be an under-recognized outcome of $A C L$ reconstruction, and be at least as common as TFJ OA.[35].. In this systematic review, the PFJ OA rate of the ipsilateral knee ranged from $28.1-80.2 \%$ and meanly at $46.8 \%$. For the contralateral side, it ranged from 9.0-56.3\% and meanly at 30.8\%. On the ipsilateral side, the risk ratio of developing OA in the TFJ was merely 1.15 compared with that in the PFJ. And compared with contralateral side, the risk ratio of developing $\mathrm{PFJ}$ radiographic OA was 2.42 on the ipsilateral knee. These outcomes indicated that we should stay vigilant of the OA developed in the PFJ on the ipsilateral side, which could result in anterior knee pain and knee function impairment long-term after ACL reconstruction [25, 26]. In this study, the PFJ radiographic OA between the ipsilateral side and contralateral side and the ipsilateral OA between the TFJ and PFJ was for the first time meta-analyzed.

There was an interesting phenomenon in the literature about the long-term clinical results of $\mathrm{ACL}$ reconstruction. Briefly, despite many described radiographic evaluation methods respectively on TFJ and PFJ, only very few of them did report the radiographic OA outcomes individually for PFJ and TFJ. Instead, the overall knee OA rates, the summarization of both $\mathrm{PFJ}$ and TFJ OA outcomes, were simply reported $[4-6,16,17,42,46]$. This rendered it difficult to perform a meta-analysis on the PFJ and the TFJ radiographic OA 


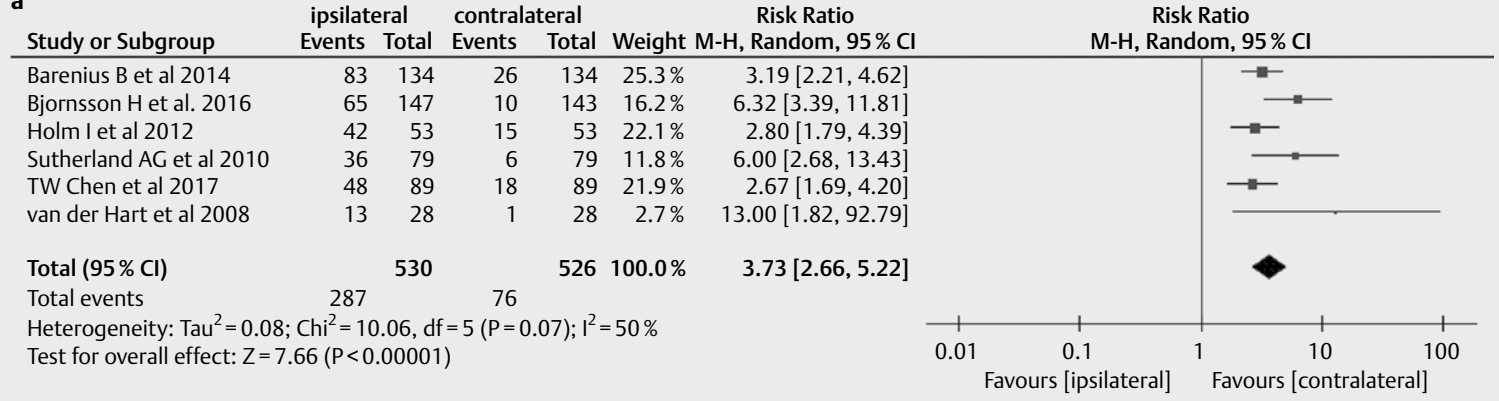

b

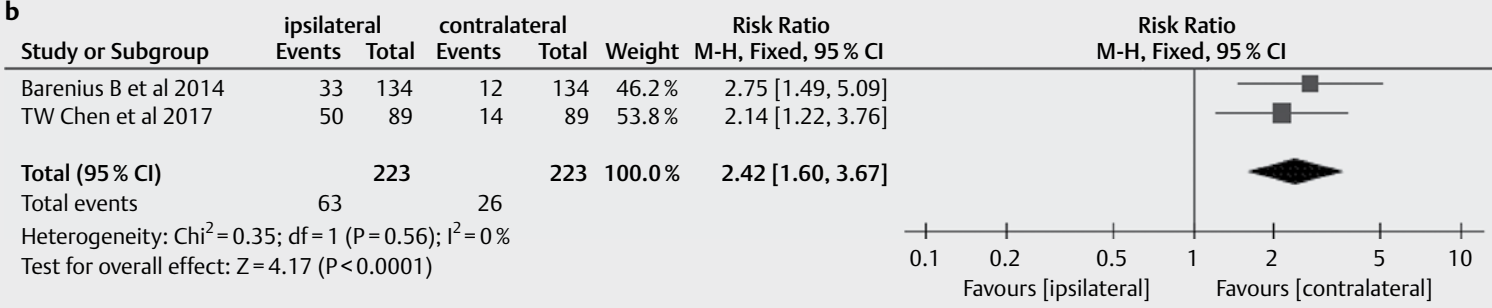

$\begin{array}{llll}\text { ipsilateral contralateral } & \text { Risk Ratio } \\ \text { Study or Subgroup } & \text { Events Total Events Total Weight } & \text { M-H, Fixed, 95\% C }\end{array}$

\begin{tabular}{lllllll}
\hline Barenius B et al 2014 & 80 & 134 & 25 & 134 & $58.1 \%$ & $3.20[2.19,4.68]$
\end{tabular}

$\begin{array}{lllllll}\text { TW Chen et al } 2017 & 44 & 89 & 18 & 89 & 41.9 \% & 2.44[1.54,3.88]\end{array}$

Total $(95 \% \mathrm{Cl})$

223

$223100.0 \%$

$2.88[2.15,3.87]$

Total events $124 \quad 43$

Heterogeneity: $\mathrm{Chi}^{2}=0.78 ; \mathrm{df}=1(\mathrm{P}=0.38) ; \mathrm{I}^{2}=0 \%$

Test for overall effect: $Z=7.08(P<0.00001)$

Risk Ratio

d

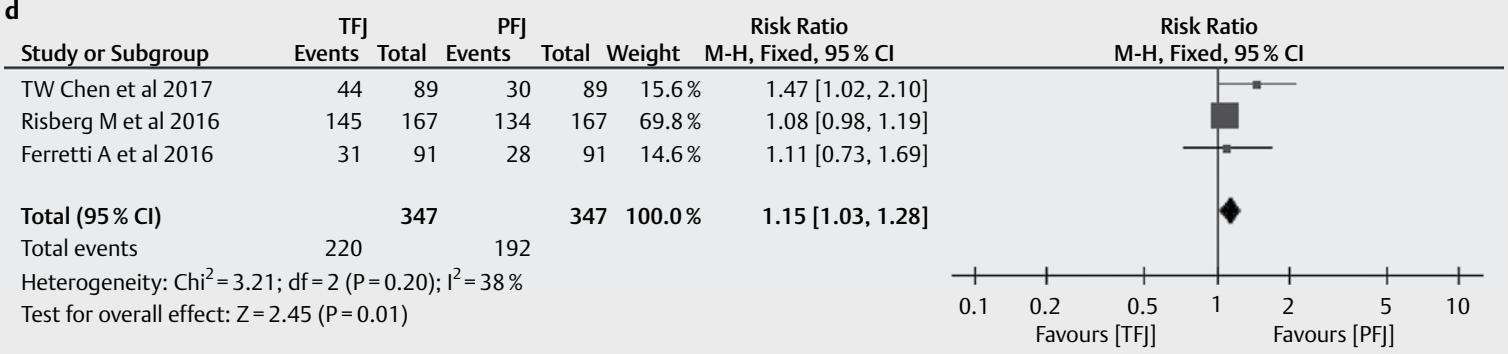

e

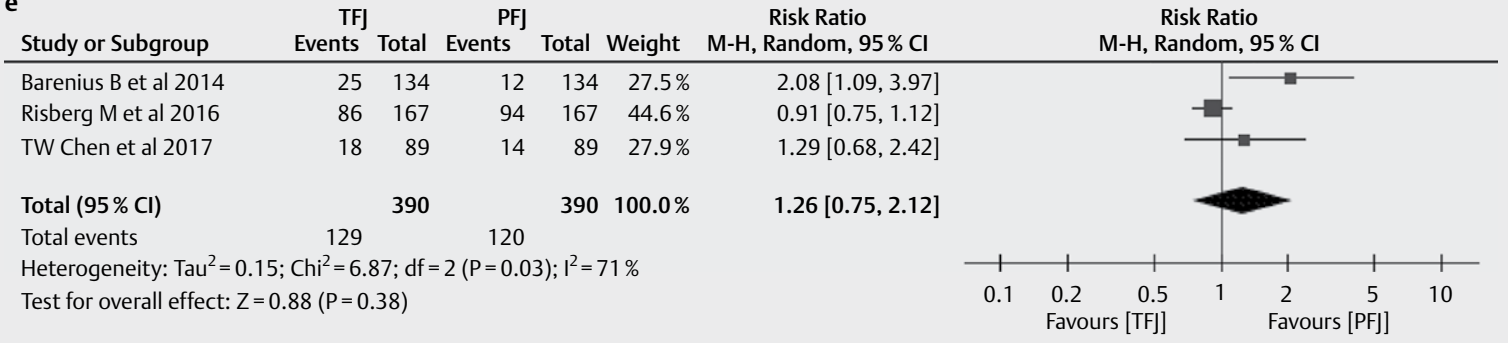

Fig. 2 a Forest plot of ipsilateral versus contralateral knees in developing overall knee radiographic OA. Risk ratio $=3.73$ (P<0.00001), with heterogeneity of $50 \%$, $(P=0.07)$; b Forest plot of ipsilateral versus contralateral knees in developing patellofemoral joint radiographic OA. Risk ratio $=2.42$ $(\mathrm{P}<0.0001)$, with heterogeneity of $0 \%,(\mathrm{P}=.56)$; c Forest plot of ipsilateral versus contralateral knees in developing tibiofemoral joint radiographic OA. Risk ratio $=2.88(\mathrm{P}<0.00001)$, with heterogeneity of $0 \%,(\mathrm{P}=.38)$; $\mathbf{d}$ Forest plot of tibiofemoral joint versus patellofemoral joint in developing radiographic $O A$ on the ipsilateral side. Risk ratio $=1.15(P=0.01)$, with heterogeneity of $38 \%$, $(P=.20)$; e Forest plot of tibiofemoral joint versus patellofemoral joint in developing radiographic OA on the contralateral side. Risk ratio $=1.26(P=0.38)$, with heterogeneity of $71 \%$, $(P=0.03)$.

after $\mathrm{ACL}$ reconstruction. In previous studies by Ajuied et al. [21], Claes et al. [20], and Oiestad et al. [19], the researchers only fulfilled the meta-analysis on the overall knee OA. As Claes et al. mentioned, "it was decided to pool all available data in one general group of 'knee OA'”. In this study, unpublished data of the TFJ and PFJ OA were shared by some researchers whose studies were therefore included in the meta-analysis, allowing further exploration. 
The methodology quality of evidence in meta-analysis is vital. In 2009, Oiestad et al. adopted the modified Coleman Methodology Score (MCMS) for the quality evaluating of included studies in their research. It was concluded that the MCMS was low for the included studies without a universal methodological radiologic classification. Thus it was difficult to compare the studies reporting the knee OA prevalence more than ten years after ACL injury, as well as to state firm conclusions on that. Herein, only the studies adopting the Kellgren\&Lawrence classification, a richly applied and widely reported grading system for knee OA evaluation, were included for analysis. Moreover, we performed the methodological quality assessment on all included studies. In 2014, Ajuied et al. reported the meta-analysis on radiographic knee OA at minimal ten years after $A C L$ injury, extracting data from the studies published before 2013. To the best of our knowledge, there has not been any metaanalysis on the topic since 2013. In the meantime, several studies on the long-term clinical outcomes of ACL reconstruction were successively reported. It should be highlighted that the average MCMS of the nine studies published between 2014 and 2017 was 10 points higher than those published earlier than 2014. Owing to the merits of better study design and standardized reporting strategy, we were able to analyzed the OA between the ipsilateral and contralateral sides, and furtherly investigated the OA between the TFJ and PFJ in this meta-analysis.

There were some limitations in this study. Firstly, only four studies reporting the TFJ or PFJ radiographic OA included in this study. Secondly, the analysis of combined injuries like meniscus or cartilage lesion herein was not performed due to insufficient data from the included studies. Thirdly, the attrition of subjects was high. In some study the radiographic evaluation was merely about $60 \%$ or even not reported. Fourthly, the response rate was lower than expected.

There were also some advantages. Firstly, the study provided an up to date, in-depth view of the long-term radiographic $O A$ at minimal ten years after $A C L$ reconstruction. Owing to better quantity and quality of the evidence, this review stated firm conclusion on the long-term OA prevalence over 10 years after $A C L$ reconstruction. Secondly, compared with previous researches, the study focused on a more specific group of the ACL reconstructed cases by excluding those conservatively treated. Thirdly, previous metaanalysis failed to investigate in detail the $\mathrm{OA}$ rate in $\mathrm{ACL}$ reconstruction, and studies of higher quality recently published made it possible to further analyze the OA prevalence long-term ACL reconstruction, especially in different joints (TFJ and PFJ). The OA in the PFJ was reportedly prevalent and associated with worse symptoms and function impairment long-term after ACL reconstruction like dramatic swelling, valgus deformity, markedly reduced quadriceps strength, and pain on the PFJ compression, while the TFJ OA were associated with bony enlargement or deformity, reduced flexion range of movement, mediolateral instability and varus deformity [49]. For the differences of symptoms and signs between the PF] $\mathrm{OA}$ and the TFJ OA, the health care providers need to remain vigilant to risk factors of both. This meta-analysis compared, for the first time, the radiographic OA of the TFJ between ipsilateral and contralateral sides, as well as that of the PFJ. Also, the study for the first time compared the radiographic OA of the PFJ and that of the TFJ, respectively, on ipsilateral and contralateral sides.

\section{Conclusion}

The current evidence suggested that the ACL reconstruction cannot spare the knee from developing radiographic OA long-term postoperative. Over a minimum of 10 years after surgery, about half the cases developed overall radiographic OA on the ipsilateral knee, which is nearly four times higher than the contralateral side. On the ipsilateral knee, the TFJ was mostly affected, which is about 1.15 times higher than the PFJ.

\section{Acknowledgements}

Much grateful for the authors who shared their precious unpublished data, they are: v Björn Barenius, Department of Clinical Science and Education, Karolinska Institutet, Stockholm, Sweden v Andrea Ferretti and Antonio Ponzo, the La Sapienza University, Sant' Andrea Hospital, Rome, Italy v Haukur Björnsson, the Department of Orthopaedics, Institute of Clinical Sciences, Sahlgrenska Academy, University of Gothenburg, Sweden v May Arna Risberg, the Department of Sport Medicine, Norwegian School of Sport Sciences, Oslo, Norway.

\section{Funding}

1) Shanghai Sailing Program (No. 19YF1414600), 2) Open-fund of State Key Laboratory of Advanced Technology for Materials Synthesis and Processing (Wuhan University of Technology) (No. 2018KF-12).

\section{Conflict of Interest}

Authors declare that they have no conflict of interest.

\section{References}

[1] Bjornsson H, Samuelsson K, Sundemo D, Desai N, Sernert N, Rostgard-Christensen L, Karlsson J, Kartus J. A randomized controlled trial with mean 16-year follow-up comparing hamstring and patellar tendon autografts in anterior cruciate ligament reconstruction. Am J Sports Med 2016; 44: 2304-2313

[2] Cohen M, Amaro JT, Ejnisman B, Carvalho RT, Nakano KK, Peccin MS, Teixeira R, Laurino CFS, Abdalla RJ. Anterior cruciate ligament reconstruction after 10-15 years: Association between meniscectomy and osteoarthrosis. Arthroscopy 2007; 23: 629-634

[3] Crawford SN, Waterman BR, Lubowitz JH. Long-term failure of anterior cruciate ligament reconstruction. Arthroscopy 2013; 29: 1566-1571

[4] Gerhard P, Bolt R, Duck K, Mayer R, Friederich NF, Hirschmann MT. Long-term results of arthroscopically assisted anatomical single-bundle anterior cruciate ligament reconstruction using patellar tendon autograft: Are there any predictors for the development of osteoarthritis? Knee Surg Sports Traumatol Arthrosc 2013; 21: 957-964

[5] Hoffelner T, Resch H, Moroder P, Atzwanger ], Wiplinger M, Hitzl W, Tauber M. No increased occurrence of osteoarthritis after anterior cruciate ligament reconstruction after isolated anterior cruciate ligament injury in athletes. Arthroscopy 2012; 28: 517-525 
[6] Leiter JR, Gourlay R, McRae S, de Korompay N, MacDonald PB. Long-term follow-up of ACL reconstruction with hamstring autograft. Knee Surg Sports Traumatol Arthrosc 2014; 22: 1061-1069

[7] Mansson O, Sernert N, Rostgard-Christensen L, Kartus J. Long-term clinical and radiographic results after delayed anterior cruciate ligament reconstruction in adolescents. Am J Sports Med 2015; 43: 138-145

[8] Meuffels DE, Favejee MM, Vissers MM, Heijboer MP, Reijman M, Verhaar JA. Ten year follow-up study comparing conservative versus operative treatment of anterior cruciate ligament ruptures. A matched-pair analysis of high level athletes. Br J Sports Med 2009; 43: 347-351

[9] Myklebust G, Holm I, Maehlum S, Engebretsen L, Bahr R. Clinical, functional, and radiologic outcome in team handball players 6-11 years after anterior cruciate ligament injury: A follow-up study. Am J Sports Med 2003; 31: 981-989

[10] Peck J. Long-term sequelae and management following anterior cruciate ligament injury. BMJ Case Rep 2014; 2014: bcr2014204239

[11] Sanders TL, Pareek A, Kremers HM, Bryan AJ, Levy BA, Stuart M], Dahm DL, Krych AJ. Long-term follow-up of isolated ACL tears treated without ligament reconstruction. Knee Surg Sports Traumatol Arthrosc 2017; 25: 493-500

[12] Spindler KP, Parker RD, Andrish JT, Kaeding CC, Wright RW, Marx RG, McCarty EC, Amendola A, Dunn WR, Huston LJ, Harrell FE Jr. MOON Group. Prognosis and predictors of $\mathrm{ACL}$ reconstructions using the MOON cohort: A model for comparative effectiveness studies. J Orthop Res 2013; 31: 2-9

[13] Lohmander LS, Englund PM, Dahl LL, Roos EM. The long-term consequence of anterior cruciate ligament and meniscus injuries: Osteoarthritis. Am J Sports Med 2007; 35: 1756-1769

[14] Pinczewski LA, Lyman J, Salmon LJ, Russell V], Roe J, Linklater J. A 10-year comparison of anterior cruciate ligament reconstructions with hamstring tendon and patellar tendon autograft: A controlled, prospective trial. Am J Sports Med 2007; 35: 564-574

[15] Risberg MA, Holm I, Steen H, Eriksson J, Ekeland A. The effect of knee bracing after anterior cruciate ligament reconstruction. A prospective, randomized study with two years' follow-up. Am J Sports Med 1999; 27: $76-83$

[16] Struewer ], Frangen TM, Ishaque B, Bliemel C, Efe T, Ruchholtz S, Ziring E. Knee function and prevalence of osteoarthritis after isolated anterior cruciate ligament reconstruction using bone-patellar tendon-bone graft: Long-term follow-up. Int Orthop 2012; 36: 171-177

[17] Falciglia F, Panni AS, Giordano M, Aulisa AG, Guzzanti V. Anterior cruciate ligament reconstruction in adolescents (Tanner stages 2 and 3). Knee Surg Sports Traumatol Arthrosc 2016; 24: 807-814

[18] Gillquist J, Messner K. Anterior cruciate ligament reconstruction and the long-term incidence of gonarthrosis. Sports Med 1999; 27 : 143-156

[19] Oiestad BE, Engebretsen L, Storheim K, Risberg MA. Knee osteoarthritis after anterior cruciate ligament injury: A systematic review. Am J Sports Med 2009; 37: 1434-1443

[20] Claes S, Hermie L, Verdonk R, Bellemans J, Verdonk P. Is osteoarthritis an inevitable consequence of anterior cruciate ligament reconstruction? A meta-analysis. Knee Surg Sports Traumatol Arthrosc 2013; 21: 1967-1976

[21] Ajuied A, Wong F, Smith C, Norris M, Earnshaw P, Back D, Davies A. Anterior cruciate ligament injury and radiologic progression of knee osteoarthritis: A systematic review and meta-analysis. Am J Sports Med 2014; 42: 2242-2252

[22] Barenius B, Ponzer S, Shalabi A, Bujak R, Norlen L, Eriksson K. Increased risk of osteoarthritis after anterior cruciate ligament reconstruction: A 14-year follow-up study of a randomized controlled trial. Am J Sports Med 2014; 42: 1049-1057
[23] Bjorkman P, Sandelin J, Harilainen A. A randomized prospective controlled study with 5-year follow-up of cross-pin femoral fixation versus metal interference screw fixation in anterior cruciate ligament reconstruction. Knee Surg Sports Traumatol Arthrosc 2015; 23: 2353-2359

[24] Culvenor AG, Collins N], Guermazi A, Cook JL, Vicenzino B, Whitehead TS, Morris HG, Crossley KM. Early patellofemoral osteoarthritis features one year after anterior cruciate ligament reconstruction: Symptoms and quality of life at three years. Arthritis Care Res (Hoboken) 2016; 68: 784-792

[25] Culvenor AG, Lai CC, Gabbe B], Makdissi M, Collins NJ, Vicenzino B, Morris HG, Crossley KM. Patellofemoral osteoarthritis is prevalent and associated with worse symptoms and function after hamstring tendon autograft ACL reconstruction. Br J Sports Med 2014; 48: 435-439

[26] Culvenor AG, Oiestad BE, Holm I, Gunderson RB, Crossley KM, Risberg MA. Anterior knee pain following anterior cruciate ligament reconstruction does not increase the risk of patellofemoral osteoarthritis at 15- and 20-year follow-ups. Osteoarthritis Cartilage 2017; 25: 30-33

[27] Ferretti A, Monaco E, Ponzo A, Basiglini L, lorio R, Caperna L, Conteduca F. Combined intra-articular and extra-articular reconstruction in anterior cruciate ligament-deficient knee: 25 years later. Arthroscopy 2016; 32: 2039-2047

[28] Peterson L, Eklund U, Engstrom B, Forssblad M, Saartok T, Valentin A. Long-term results of a randomized study on anterior cruciate ligament reconstruction with or without a synthetic degradable augmentation device to support the autograft. Knee Surg Sports Traumatol Arthrosc 2014; 22: 2109-2120

[29] Risberg MA, Oiestad BE, Gunderson R, Aune AK, Engebretsen L, Culvenor A, Holm I. Changes in knee osteoarthritis, symptoms, and function after anterior cruciate ligament reconstruction: A 20-Year Prospective Follow-up Study. Am J Sports Med 2016; 44: 1215-1224

[30] Ruffilli A, Buda R, Pagliazzi G, Baldassarri M, Cavallo M, Luciani D, Ferranti E, Giannini S. Over-the-Top anterior cruciate ligament reconstruction using single- or double-strand hamstrings autograft. Orthopedics 2015; 38: e635-e643

[31] Tengman E, Brax Olofsson L, Nilsson KG, Tegner Y, Lundgren L, Hager CK. Anterior cruciate ligament injury after more than 20 years: I. Physical activity level and knee function. Scand J Med Sci Sports 2014; 24: e491-e500

[32] Tengman E, Brax Olofsson L, Stensdotter AK, Nilsson KG, Hager CK. Anterior cruciate ligament injury after more than 20 years. II. Concentric and eccentric knee muscle strength. Scand J Med Sci Sports 2014; 24: e501-e509

[33] Thompson S, Salmon L, Waller A, Linklater J, Roe J, Pinczewski L. Twenty-year outcomes of a longitudinal prospective evaluation of isolated endoscopic anterior cruciate ligament reconstruction with patellar tendon autografts. Am J Sports Med 2015; 43: 2164-2174

[34] Webster KE, Feller JA, Hartnett N, Leigh WB, Richmond AK. Comparison of patellar tendon and hamstring tendon anterior cruciate ligament reconstruction: A 15-year follow-up of a randomized controlled trial. Am J Sports Med 2016; 44: 83-90

[35] Culvenor AG, Cook JL, Collins NJ, Crossley KM. Is patellofemoral joint osteoarthritis an under-recognised outcome of anterior cruciate ligament reconstruction? A narrative literature review. $\mathrm{Br}$ J Sports Med 2013; 47: 66-70

[36] Harriss D], Macsween A, Atkinson G. Standards for ethics in sport and exercise science research: 2018 update. Int J Sports Med 2017; 38: 1126-1131

[37] Coleman BD, Khan KM, Maffulli N, Cook JL, Wark JD. Studies of surgical outcome after patellar tendinopathy: Clinical significance of methodological deficiencies and guidelines for future studies. Victorian Institute of Sport Tendon Study Group. Scand J Med Sci Sports 2000; 10: 2-11 
[38] Tallon C, Coleman BD, Khan KM, Maffulli N. Outcome of surgery for chronic Achilles tendinopathy. A critical review. Am J Sports Med 2001; 29: $315-320$

[39] Shrier I, Boivin JF, Steele RJ, Platt RW, Furlan A, Kakuma R, Brophy ], Rossignol M. Should meta-analyses of interventions include observational studies in addition to randomized controlled trials? A critical examination of underlying principles. Am J Epidemiol 2007; 166: 1203-1209

[40] Chen T, Zhang P, Chen J, Hua Y, Chen S. Long-term outcomes of anterior cruciate ligament reconstruction using either synthetics with remnant preservation or hamstring autografts: A 10-year longitudinal study. Am J Sports Med 2017; 45: 2739-2750

[41] Holm I, Oiestad BE, Risberg MA, Gunderson R, Aune AK. No differences in prevalence of osteoarthritis or function after open versus endoscopic technique for anterior cruciate ligament reconstruction: 12-year follow-up report of a randomized controlled trial. Am J Sports Med 2012; 40: 2492-2498

[42] Janssen RP, du Mee AW, van Valkenburg J, Sala HA, Tseng CM. Anterior cruciate ligament reconstruction with 4-strand hamstring autograft and accelerated rehabilitation: A 10-year prospective study on clinical results, knee osteoarthritis and its predictors. Knee Surg Sports Traumatol Arthrosc 2013; 21: 1977-1988

[43] Mascarenhas R, Tranovich M, Karpie JC, Irrgang JJ, Fu FH, Harner CD. Patellar tendon anterior cruciate ligament reconstruction in the high-demand patient: Evaluation of autograft versus allograft reconstruction. Arthroscopy 2010; 26: (9 Suppl): S58-S66
[44] Seon JK, Song EK, Park SJ. Osteoarthritis after anterior cruciate ligament reconstruction using a patellar tendon autograft. Int Orthop 2006; 30: 94-98

[45] Sutherland AG, Cooper K, Alexander LA, Nicol M, Smith FW, Scotland $T R$. The long-term functional and radiological outcome after open reconstruction of the anterior cruciate ligament. J Bone Joint Surg Br 2010; 92: 1096-1099

[46] van der Hart CP, van den Bekerom MP, Patt TW. The occurrence of osteoarthritis at a minimum of ten years after reconstruction of the anterior cruciate ligament. J Orthop Surg Res 2008; 3: 24

[47] Harris KP, Driban JB, Sitler MR, Cattano NM, Balasubramanian E, Hootman JM. Tibiofemoral osteoarthritis after surgical or nonsurgical treatment of anterior cruciate ligament rupture: A systematic review. J Athl Train 2017; 52: 507-517

[48] Oiestad BE, Holm I, Engebretsen L, Aune AK, Gunderson R, Risberg MA. The prevalence of patellofemoral osteoarthritis 12 years after anterior cruciate ligament reconstruction. Knee Surg Sports Traumatol Arthrosc 2013; 21: 942-949

[49] Peat G, Duncan RC, Wood LR, Thomas E, Muller S. Clinical features of symptomatic patellofemoral joint osteoarthritis. Arthritis Res Ther 2012; 14: R63 